\title{
The decentralization of a sales support department in a medium-large company
}

\section{A quantitative assessment based on ideas of Thomas L. Saaty and Stafford Beer}

\author{
A.E. Steenge, A. Bulten and F.G. Peters \\ University of Twente, Faculty of Public Administration, P.O. Box 217, 7500 AE Enschede, The Netherlands
}

\begin{abstract}
In this paper we discuss an integration of the Beer cybernetic oriented management theory and Saaty's hierarchical approach to assess management problems in a more quantitative way.
\end{abstract}

Keywords: Decentralisation, hierarchical decomposition, information flow, synergy, cybernetics

\section{Introduction}

In present-day management literature, cybernetics-based system-oriented thinking has firmly established a place of its own. In this branch of literature, a socio-economic system is thought of as an organism having a number of well-specified functions, striving to survive in an unsure and potentially hostile environment. Well-known authors in this tradition are Beer $(1966,1979)$, Aldrich (1979), Weick (1979), Mintzberg (1979, 1983), Gomez (1981), Scott (1981), Kieser and Kubicek (1983), and Levinson (1985).

A primary concept of organization here is the system's capability of self-regulation in terms of externally defined states of the environment. This requires that essential system parameters be kept within certain well-defined limits to prevent the system from collapsing. If these limits are observed, the system not only survives, but, as formulated by Stafford Beer (1979, p. 113), we may speak of 'survival in reasonable comfort'.

Beer's management cybernetics theory focuses on the self-regulating and self-organizing powers of a system that make the system's development and behaviour relatively invariant to specific ac-

Received November 1989 tions and interventions from outside (see also Gomez, 1981). As a central concept, Beer has introduced the viable system. Viable systems are able to maintain their identities in an environment known for - often - unpredictable action and behaviour.

The structure of a viable system is characterized by a hierarchy of sub-systems that can be identified with the following system functions (Gomez, 1981, p. 87 ff.). See Figure 1.

1. System 1 (the divisional function) consists of relatively independent operational units (the divisions) performing according to the firm's general objectives. An example of this system is the work floor, where the actual products are being manufactured.

2. System 2 is a functional system where a specific kind of coordination (both vertical and horizontal) takes place between separate operational units. The central concept here is 'damping'. Each division is autonomous within the limits set by the organization, but this autonomy can be seriously disrupted by other units, e.g., because of communication failures or conflicts of interests. To prevent it, System 2 coordinates the divisions.

3. System 3 (the operational function) ensures the internal stability within the firm. It aims at providing an optimal distribution and use of avail- 


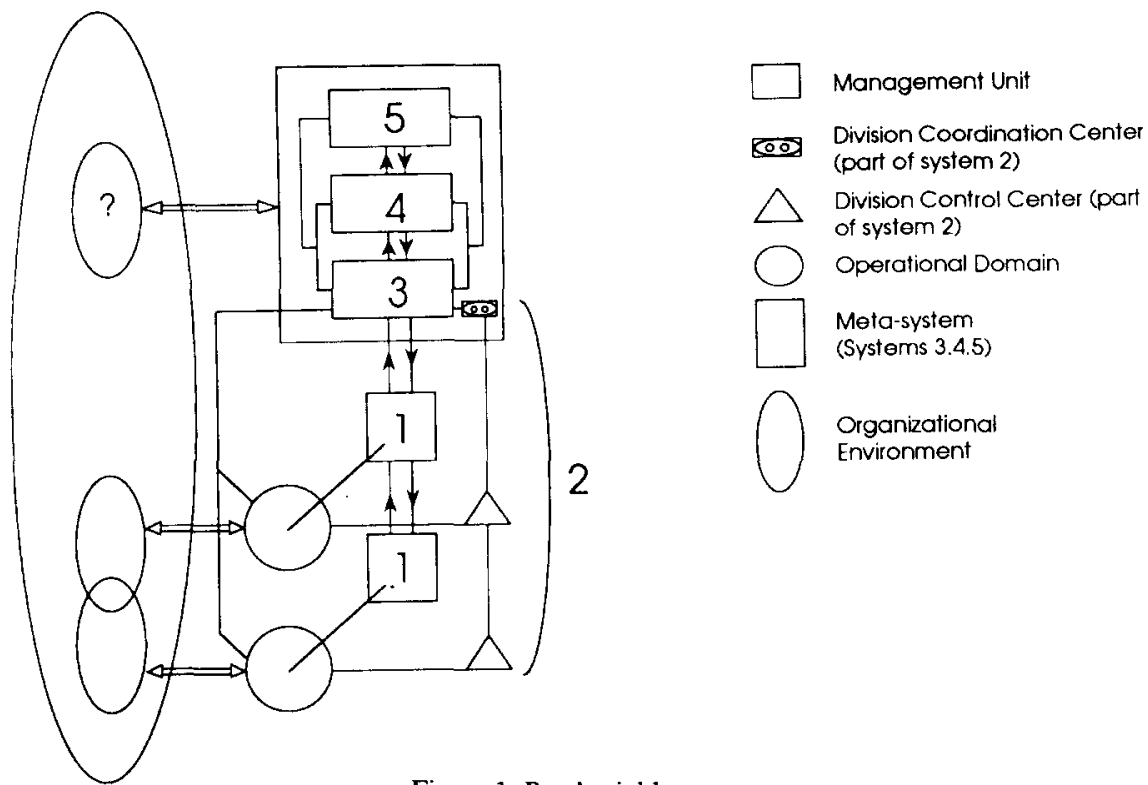

Figure 1. Beer's viable system

able resources and a proper functioning of activities within the firm.

4. System 4 (the strategic function) guarantees the stability of the firm with respect to its environment. The main purpose of this system is to ensure continuity.

5. System 5 (the normative function) coordinates Systems 3 and 4, in accordance with the firm's overall policy. It seeks to balance external and internal stability.

These five system functions should not be identified a priori with one particular person or group of persons (although, for example, the general manager may represent the System 3 function). They rather represent groups of activities which can be identified throughout each (sub-) system. In the case we shall discuss in some detail in this paper, attention will be focused on the system functions 1 and 2. Therefore, in order not to complicate the analysis unnecessarily, Systems 3, 4 and 5 have been lumped together in one - aggregated - function, henceforth to be called the "Metasystem".

The above-mentioned functions are necessary to a system's viability, but they are not sufficient. Beer has formulated three further principles of organizational effectiveness (Beer, 1979): Requisite variety, Channel capacity, and Transduction.

\section{The planned reorganization}

The subject of our inquiry is a Dutch company that produces capital goods for the use and distribution of electric energy. In the 1960's, six major companies in Holland merged into this company. In this process, the sales departments were centralized. This was done primarily to prevent a potential client from being visited by several salesmen of the same company within a short period of time.

At present the company's primary processes take place in the so-called "Industry Groups". The planned reorganization of one of the three groups which exist at the moment will be discussed in this paper. Within this particular industry group three 'Product Groups' can be distinguished. Production methods of the different product groups may differ substantially. However, often complementary products ask for combined orders for all three product groups.

The essential task of the Central Sales Support Department is assuring smooth connections between the commercially oriented sales departments and the technically oriented production units. Taking care of the combined orders is one of the three main activities of Central Sales Support. Its other main activities are the 'normal' sales support activities (preparing quotations or 




Figure 2. Central sales support as a synergetic coordination function

preparing orders for production) and the so-called "central activities", primarily the representation of the interests of Central Sales Support at the sales and production branches.

In the planned reorganization, the (single) industry group will be split into three new Industry Groups. In this process, Central Sales Support will be decentralized. Of the original function, only an administrative top remains, which is responsible for a number of staff activities which cannot be assigned directly.

The decentralization of the industry group is a vertical one (Mintzberg, 1983), the delegation of decision power going down. The decentralization of the Central Support Department is partly a vertical decentralization (the former product groups become responsible for the assigned sales support) and partly just a physical distribution of certain services, means and people, where decision power is not involved.
The translation of the organizational chart at $t 1$ - the situation before decentralization - into the terminology of the Beer framework is given in Figure 2. The situation after decentralization is given in Figure 3.

The subject proper of this paper concerns the effects of the planned decentralization of the Central Sales Support Department. Central here is the question if the new structure ( 12 ) will be able to provide the same support facilities to the company as before. Certain consequences of applying the Beer model to the planned decentralization procedure are immediately obvious. For example, looking at the second translation (Figure 3), it is clear that the synergetic effect of the (original) sales support is largely lost: the lines of communication between Sales and Sales Support do not conform to Beer's organization model. It is also clear that the relation between the new sales support departments is not well-organized. This is 


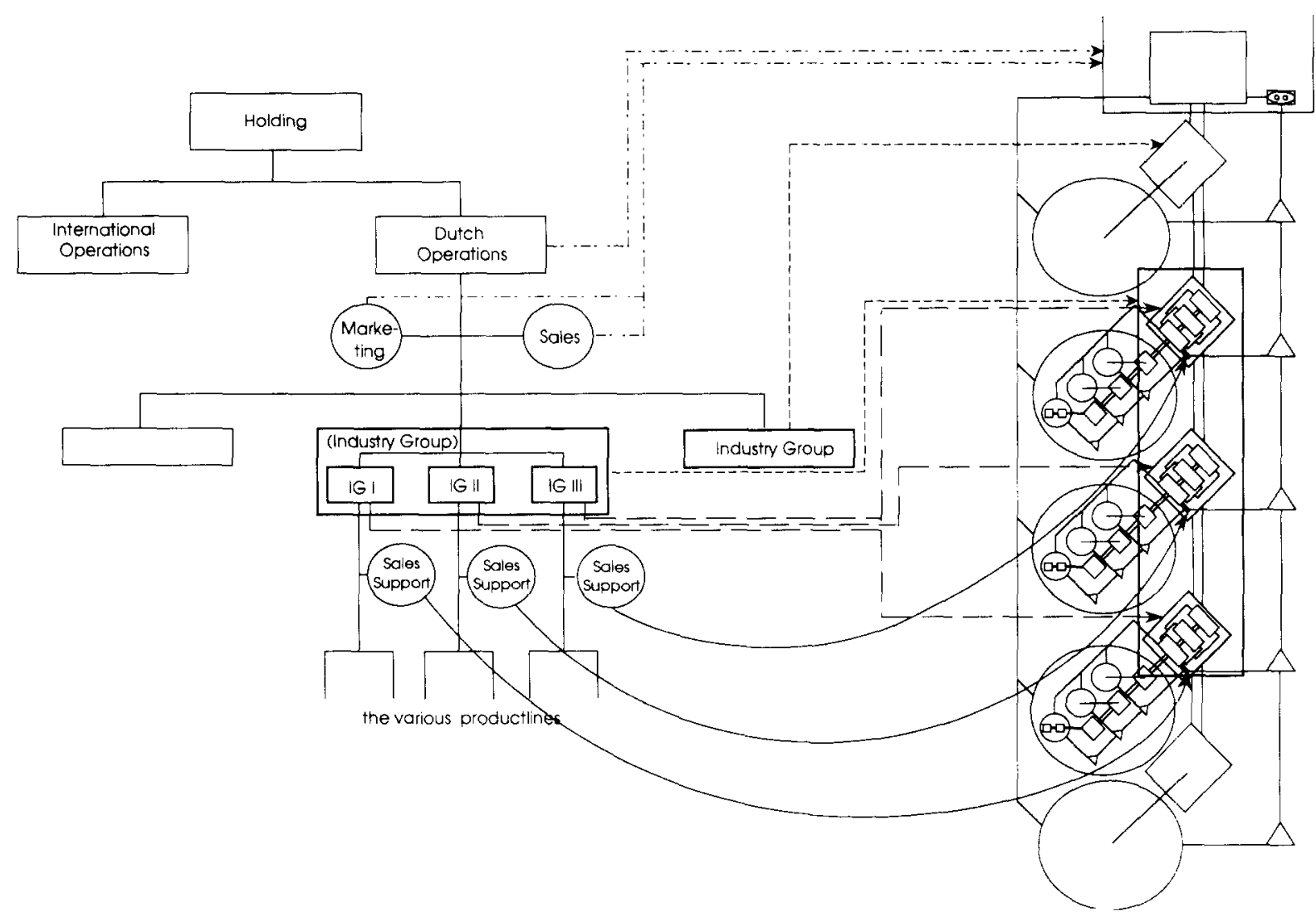

Figure 3. Central sales support departments lacking synergetic links

illustrated by Figure 4, which is an enlargement of the small rectangular area at the right-hand part of Figure 3. An immediate consequence of this will be that combined orders may be expected to be more difficult to process in the new situation.

Because of the many separate issues to be considered, a total verdict on the integral decentrali-

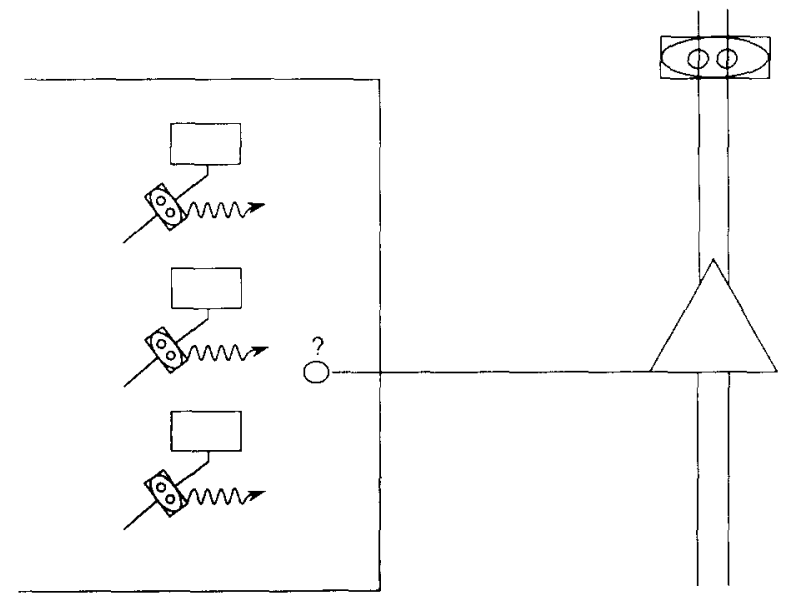

Figure 4. Ill-organized communication zation operation is difficult to give without a quantitative approach. Although the Beer model allows numerical calculations, the Saaty hierarchical approach probably will be most helpful in translating the effects of the (proposed) changes into numerical values (Saaty, 1980; Saaty and Alexander, 1981). A prominent reason is that the two situations (before and after the decentralization) have to be compared on each organizational level. For work of such scope, the AHP seems preferable to others. Our paper thus, will concentrate on a method to compare existing (t1) and the planned ( $\mathrm{t} 2$ ) organizational framework. In a final section we discuss some possibilities regarding the location and elimination of organizational bottlenecks.

\section{The hierarchy}

In this section, we consider a well-functioning Sales Support effort as the central aim. In the t1-situation, the Central Sales Support Depart- 


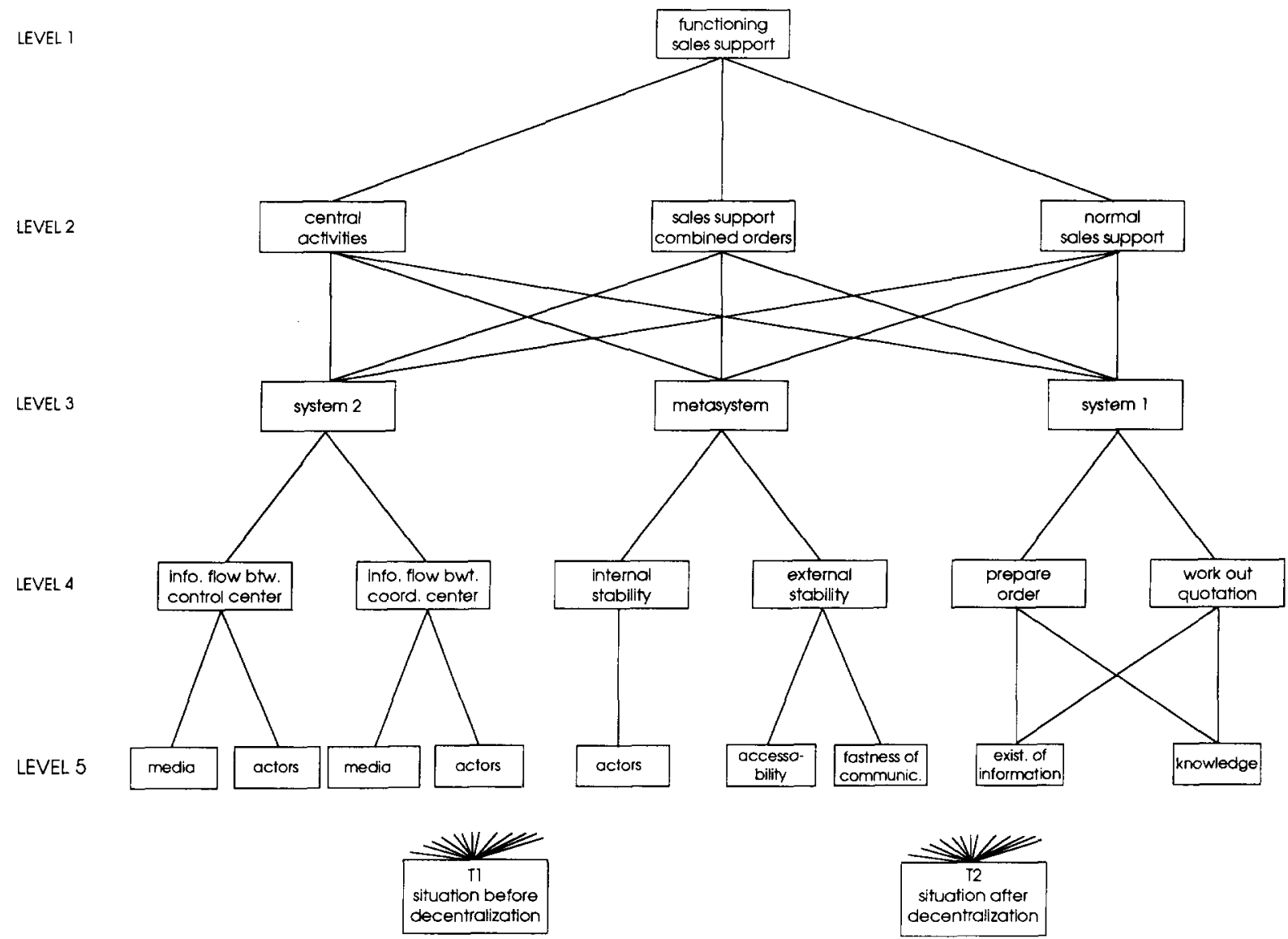

Figure 5. Hierarchical decomposition of the function of sales support

ment (CSS, see Figure 2) is the central unit. We already briefly mentioned that it has three main tasks, i.e.:

(1) General activities, mainly taking care of the CSS-interests.

(2) 'Normal' sales support activities, where only one product group is involved.

(3) Sales support activities involving combined orders. As we have seen, in the t2-situation, CSS is split into three new support groups (see the lefthand side of Figure 3).

For sales support to contribute to the viability of the system in the Beer cybernetic interpretation, its System 1, System 2, and Metasystem functions must perform well, and be well coordinated. The cybernetic structure can be numerically analyzed by considering to what extent each institution contributes to each system function. Diagrammatically, we have the configuration of Figure 5. For reasons of space, in this paper, we shall concentrate mainly on the System 2 function.
The levels have the following interpretation:

Level 1. Overall performance of the Sales Support organization (SS).

Level 2. The institutions (groups of activities): Central Activities (CA), Sales Support of Combined Orders (SSCO), Normal Sales Support (NSS).

Level 3. The contributions to the several system functions we have distinguished: System 1 (S1), System 2 (S2), and the Metasystem function (M), which combines the functions of the system functions 3, 4 and 5 we discussed above.

Level 4. This level consists of three groups of factors, each associated with a system function. For System 2, we distinguish the flow of information between the various control centers (CONT), and the flow of information between the various coordination centers (COORD). For the Metasystem we distinguish internal and external stability as factors, and for System 1 the processing of, respectively, orders and quotations. 
Level 5. On this level we distinguish means of communication (Media), number of people involved (Actors), Accessability, speed of Communication, Existence and quality of information and the available Knowledge and experience. From Figure 5 we immediately have that the terms 'Media' and 'Actors' have a different interpretation in each system function.

Level 6 . The situation before ( $\mathrm{t} 1)$ and after $(\mathrm{t} 2)$ the decentralization effort.

Our hierarchy thus consists of 6 levels. If we want to incorporate additional detail, we may wish to take even higher levels into consideration. For example, the factors Media and Actors can be analyzed in terms of the criteria 'type' and 'size'. In order not to complicate the analysis unnecessarily, we have omitted such higher levels. Because not every criterium at the higher levels affects each criterium on the previous level, the hierarchy should be characterized as an incomplete one, in the sense defined by Saaty (1990, p. 42-43). This means that at the appropriate places, priorities and their reciprocals have been represented by a zero.

\section{Results}

First of all, we have to estimate the impact of the three institutions or groups of activities (i.e. CA, SSCO, NSS) on the overall functioning of the company's sales support effort. In the comparison matrix giving these impacts, the interests of all concerned parties (the Sales, Sales Support, Production, and other Departments) should be reflected. On the basis of interviews, questionnaires and various kinds of reports, the following pairwise comparison matrix was compiled for SS (Level 1) with $a$ its eigenvector:

$$
\operatorname{SSCO}\left(\begin{array}{ccc}
1 & \frac{1}{2} & \frac{1}{3} \\
2 & 1 & \frac{1}{2} \\
\operatorname{NSS} & 2 & 1
\end{array}\right), \quad a=\left(\begin{array}{l}
0.163 \\
0.297 \\
0.540
\end{array}\right)
$$

The next step is the determination of the system functions $\mathrm{S} 2, \mathrm{M}$ and $\mathrm{S} 1$, as they are embodied in the Level 2 groups of activities. Our inquiries lead to the following matrices, respectively, for CA:

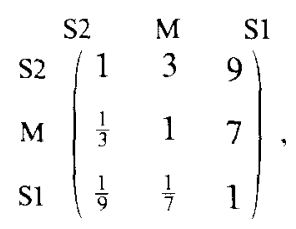

for SSCO:

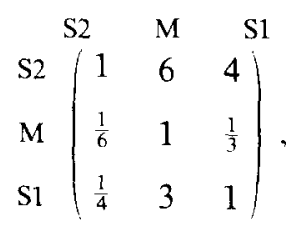

and for NSS:

$$
\mathrm{S} 2\left(\begin{array}{lll}
1 & 1 & \frac{1}{9} \\
1 & 1 & \frac{1}{7} \\
\mathrm{~S} 1 & 7 & 1
\end{array}\right) \text {. }
$$

\begin{tabular}{|c|c|c|c|}
\hline & $\begin{array}{l}\mathrm{CA} \\
0655\end{array}$ & SSCO & $\begin{array}{r}\text { NSS } \\
096\end{array}$ \\
\hline & $0>0$ & 0091 & 0105 \\
\hline & $0.2>0$ & & \\
\hline S2 & 0.055 & 0.218 & 0.799 \\
\hline
\end{tabular}

The matrix of eigenvectors, $b$, for these matrices is given by

The product $b a$ yields the vector $c$ :

$$
\mathrm{S} 2\left(\begin{array}{c}
0.364 \\
\mathrm{M} \\
\mathrm{S} 1 \\
0.131 \\
0.505
\end{array}\right) \text {. }
$$

The vector $c$ thus gives the priorities of the system functions for the well-functioning of the sales support effort. To save space, for the next levels we shall present the calculations only for the System 2 function. As indicated, CONT here stands for the information flow between the various control centers, and COORD for the information flow between the various coordination centers.

First we must determine the relative impacts of both criteria on S2 (Level 3). 
We have for $\mathrm{S} 2$

$$
\begin{aligned}
& \text { CONT COORD } \\
& \text { COORD } \quad\left(\begin{array}{ll}
1 & 3 \\
\frac{1}{3} & 1
\end{array}\right) \quad, \quad d=\left(\begin{array}{l}
0.75 \\
0.25
\end{array}\right),
\end{aligned}
$$

where $d$ is the corresponding eigenvector. For both criteria, the means of communication (meetings, memo's, telephone cables, etc.) and the number of persons involved must be considered. Using the abbreviations 'MED' and 'ACT', respectively, for the factors 'Media' and 'Actors', we have, for the first criterium (CONT):

$$
\operatorname{MED}\left(\begin{array}{ll}
1 & \frac{1}{2} \\
2 & 1
\end{array}\right), e=\left(\begin{array}{l}
0.333 \\
0.667
\end{array}\right),
$$

with $e$ the relevant eigenvector. The next level gives the direct confrontation between $\mathrm{t} 1$ and $\mathrm{t} 2$ for MED,

$$
\mathrm{t} 2\left(\begin{array}{cc}
1 & 3 \\
\frac{1}{3} & 1
\end{array}\right)
$$

and for ACT:

$$
\mathrm{t} 1\left(\begin{array}{ll}
1 & 1 \\
1 & 1
\end{array}\right) \text {. }
$$

Thus, for CONT, the matrix of eigenvectors, $f$, is given by

$$
\text { t1 }\left(\begin{array}{cc}
\text { MED } & \text { ACT } \\
\text { t2 } & 0.5 \\
0.25 & 0.5
\end{array}\right) \text {. }
$$

The product $f e$ yields the vector $g$ :

$$
\text { t1 }\left(\begin{array}{c}
\text { CONT } \\
0.583 \\
0.417
\end{array}\right) \text {. }
$$

For COORD, following the same numbers of steps, the overall priority vector $h$ is found to be

$$
\begin{aligned}
& \text { COORD } \\
& { }_{12}\left(\begin{array}{l}
0.80 \\
0.20
\end{array}\right) \text {. }
\end{aligned}
$$

Thus, the matrix of eigenvectors $i$ is given by
CONT COORD
t2 $\left(\begin{array}{ll}0.583 & 0.80 \\ 0.417 & 0.20\end{array}\right)$,

and the product id gives the priority vector for the S2 function:

$$
\text { t1 }\left(\begin{array}{c}
\text { S2 } \\
\text { t2 }(637 \\
0.363
\end{array}\right) \text {. }
$$

In similar ways, we have calculated for the Metasystem function, the eigenvector

$$
\text { t1 }\left(\begin{array}{c}
0.90 \\
0.10
\end{array}\right) \text {. }
$$

The above rather extreme result is caused by the fact that combined orders have been more or less 'forgotten' in the decision process that lead to the decentralization of the industry group and the sales support department. For the S1 vector we obtained

$$
\text { เ1 }\left(\begin{array}{c}
\text { S1 } \\
0.46 \\
0.54
\end{array}\right) \text {. }
$$

This gives, for all functions combined, the overall matrix of eigenvectors $k$ :

$$
\text { t1 }\left(\begin{array}{ccc}
\mathrm{S} 2 & \mathrm{M} & \mathrm{S} 1 \\
0.637 & 0.90 & 0.46 \\
0.363 & 0.10 & 0.54
\end{array}\right) \text {. }
$$

The product $k c$ gives the priority vector for the total sales support effort:

$$
\text { t1 } \left.\begin{array}{c}
\text { SS } \\
\text { t2 } \\
0.62 \\
0.38
\end{array}\right) \text {. }
$$

This vector gives the overall verdict on the decentralization process. We observe that after decentralization, the support function will be less effective than before. To a large extent, this is a consequence of such systematic factors as a diminished transducer variety, a loss in channel capacity and the like.

\section{Further comments}

The problem discussed in this study - the effects of the decentralization of the Central Sales Support Department in a medium-large company - can be approached in several ways. The choice 
will depend on our insights and on the possibilities we have, financially and in time. In our study, we have combined an organizational point of view with systemic functions as suggested by the viable system model. In a more extended study by one of the authors (Bulten, 1988) the comparison following a more traditional organizational point of view was carried out. Outcomes of the various comparisons were very close. Again, the pre-organization form was preferred as indicated by the vector of priorities:

$\left(\begin{array}{l}0.66 \\ 0.34\end{array}\right)$ as compared to $\left(\begin{array}{l}0.62 \\ 0.38\end{array}\right)$

obtained above.

In this paper we have shown that the overall situation before decentralization is-from the point of view of Stafford Beer's viable system theory-preferable to the situation after decentralization. A quite different question, however, is how the organization, given the new situation, should organize itself to approach the level of performance of the first (i.e., the pre-decentralization) situation. Here the concept of the system's 'capability' (the potential state of the system) should be addressed (Beer, 1979, p. 292). Fortunately, we can use the AHP and the same data gathered in the above study for this purpose.

It is not difficult to see that the coefficients of the many required pairwise comparison matrices are indicators of real and potential bottlenecks. Therefore, alternatives can be formulated to meet the requirements specified by principles of the viable-system model.
The Analytic Hierarchy Process thus enables us to measure the desirability of a formulated alternative with respect to the level of functioning of the sales support department(s). From this we can recommend a new design so that the required level of performance is reached.

\section{References}

Aldrich, H.E. (ed), Organizations and Environments, PrenticeHall, Englewood Cliffs, NJ, 1979.

Beer, St., Decision and Control: The Meaning of Operational Research and Management Cybernetics, Wiley, New York; 1966.

Beer, St., The Heart of Enterprise, Wiley, New York, 1979.

Bulten, A., "An investigation into the consequences of the decentralization of a Central Sales Support Department of a Dutch manufacturing company; Consequences and alternatives", University of Twente, Faculty of Public Administration, 1988 (in Dutch, mimeo).

Gomez, P., Modelle und Methoden der systemorientierten Management, Verlag P. Haupt, Bern, 1981.

Kieser, A., and Kubicek, H., Organisations, W. de Gruyter, Berlin, 1983.

Levinson, H., Organizational Diagnosis, Cambridge, MA, 1985.

Mintzberg, H., Structure in Five, Designing Effective Organizations, Prentice-Hall, Englewood Cliffs, NJ, 1983.

Saaty, T.L., The Analytic Hierarchy Process: Planning, Priority Setting and Resource Allocation. McGraw-Hill. New York, 1980.

Saaty, T.L., and Alexander, J.M., Thinking with Models, Pergamon Press, Oxford, 1981.

Scott, R., Organizations: Rational, Natural and Open Systems, Prentice-Hall, Englewood Cliffs, NJ, 1981.

Weick. K.E., The Social Psychology of Organizing, AddisonWesley, Reading, MA, 1979. 